Western North American Naturalist 68(3), (C) 2008, pp. 319-325

\title{
DO YELLOW-PINE CHIPMUNKS PREFER TO RECOVER THEIR OWN CACHES?
}

\author{
Stephen B. Vander Wall ${ }^{1,2}$, Cynthia J. Downs ${ }^{1}$, Mark S. Enders ${ }^{1,3}$, and Ben A. Waitman ${ }^{1,4}$
}

\begin{abstract}
Many rodent species scatter hoard seeds within a home range shared with other seed-caching animals. An animal foraging for cached food is likely to encounter the caches of other animals, as well as its own. This study asks the following question: do animals recover primarily their own caches, do they conserve their own caches and search for the caches of other animals, or do they search indiscriminately for cached seeds regardless of who buried them? We tested these ideas using 8 yellow-pine chipmunks inside rodent-proof enclosures. We allowed each subject to cache radioactive seeds, we located the seed caches, and then we paired each rodent cache with an artificial cache in a similar microsite. During search trials, 7 subjects removed mostly their own caches, and 1 subject removed similar numbers of its own caches and paired artificial caches. These results suggest that most yellow-pine chipmunks actively search for food that they have stored, apparently using spatial memory rather than relying on olfaction. However, previous studies have established that chipmunks also are very effective pilferers of caches made by other animals. This apparent discrepancy may result from a difference between enclosures and field sites in the proportion of "own" caches to total caches.
\end{abstract}

Key words: cache pilfering, hoarding, Tamias amoenus.

Many species of rodents, such as fox squirrels (Sciurus niger), Merriam's kangaroo rats (Dipodomys merriami) and yellow-pine chipmunks (Tamias amoenus), scatter hoard food within their home ranges and, in most cases, this hidden food is not actively defended by the cache maker (Stapanian and Smith 1978, Daly et al. 1992, Vander Wall 1992). Furthermore, since the home ranges of many individuals overlap, caches made by 1 individual are interspersed among caches of the population of rodents inhabiting that area. It is well known that when individuals of these species forage, they frequently encounter and pilfer caches made by other individuals (Kraus 1983, Vander Wall 2000, Leaver and Daly 2001). When cached food and caching sites are similar, members of 1 species can even pilfer the caches made by other species (Vander Wall 2000, Leaver and Daly 2001). Vander Wall and Jenkins (2003) have suggested that, for many species, pilferage of an individual's caches is almost inevitable over the long term. Individuals of these species counteract the negative effect of competition for cached food by pilfering, in turn, the caches of other individuals.

This study examines how an individual forages in a landscape containing caches made by itself and by other animals. Three possibilities exist. First, an individual could search for caches that it had made itself (using its spatial memory) but also pilfer whatever other caches it might encounter (i.e., individuals would appear to prefer foraging for their own caches). Second, an individual could ignore its own caches (by recognizing them as its own and conserving them for future use) while actively searching for (using olfaction) and pilfering the caches of other individuals. The forager could scatter hoard pilfered food items elsewhere or simply eat them. This foraging strategy might help an individual gain a competitive advantage over conspecifics and even over other species. Third, an individual could forage using only olfaction, harvesting whatever stored seeds it might encounter. The pattern of cache recovery that an individual exhibits could help reveal what information (e.g., odor cues, spatial memory) it uses while foraging and could help us better understand how individuals compete for stored food.

Yellow-pine chipmunks are ideal models for testing these ideas. They are avid scatter hoarders of seeds of pines and shrubs in dry forests of the northwestern United States and southwestern Canada. Each individual makes

\footnotetext{
${ }^{1}$ Department of Biology and the Program in Ecology, Evolution and Conservation Biology, University of Nevada, Reno, NV 89557.

2E-mail: sv@unr.nevada.edu

3E-mail: menders@unr.edu

ㅌE-mail:bawaitman@hotmail.com
} 
thousands of caches each year and scatters the caches widely throughout its home range (Kuhn and Vander Wall in press). Caches typically consist of several seeds buried $\sim 1 \mathrm{~cm}$ deep. The home ranges of dozens of yellow-pine chipmunks can overlap in a single area, meaning that the caches of 1 individual are mixed among the caches of the population at large, as well as among those of other scatter-hoarding taxa, such as long-eared chipmunks (Tamias quadrimaculatus), deer mice (Peromyscus maniculatus), Steller's Jay (Cyanocitta stelleri) and Clark's Nutcrackers (Nucifraga columbiana). Yellow-pine chipmunks can use spatial memory to retrieve caches (Vander Wall 1991) but also are very effective pilferers of the caches of other animals when the soil is moist (Vander Wall 2000, Thayer and Vander Wall 2005). Moist seeds give off a far greater signal of volatile chemical compounds and are far more likely to be discovered by olfaction (Vander Wall 1998). Seeds stored during summer and autumn are used to construct the winter larder, which is critical to winter survival (Kuhn and Vander Wall in press). Thus, the ability of individual yellowpine chipmunks to manage seeds by caching them and by pilfering them from other individuals should have strong effects on fitness.

\section{MethodS}

We conducted this study in the Whittell Forest, a field station of the Nevada System of Higher Education, $30 \mathrm{~km}$ south of Reno, Nevada $\left(39^{\circ} 15^{\prime} 10^{\prime \prime} \mathrm{N}, 119^{\circ} 52^{\prime} 35^{\prime \prime} \mathrm{W}, 1975 \mathrm{~m}\right.$ elevation). The vegetation of the site was open Jeffrey pine (Pinus jeffreyi) forest with an understory of antelope bitterbrush (Purshia tridentata). Soil consists of friable decomposed granite (for a more detailed description of the study site see Vander Wall 1998).

All experiments occurred inside 1 of three $10 \times 10$-m rodent-proof enclosures. Walls of the enclosures were constructed from 12-mm wire mesh on a wooden frame extending $\sim 75 \mathrm{~cm}$ above ground and $\sim 45 \mathrm{~cm}$ below ground. Metal flashing $20 \mathrm{~cm}$ wide lined the top of the walls both inside and outside to prevent passage of climbing rodents. Near the middle of each enclosure we buried a 20-L plastic bucket that served as a temporary refuge for subjects. The lid of the bucket was level with the ground surface, and the interior was partitioned into 3 interconnected chambers. The up- permost chamber was connected to the surface by a nearly horizontal segment of PVC pipe $\sim 50 \mathrm{~cm}$ long and $31 \mathrm{~mm}$ in diameter. All subjects readily accepted the buckets as refuge burrows.

We tested 8 subjects. To initiate a trial, we captured an adult yellow-pine chipmunk and released it into a refuge bucket. Then we placed 150 color-marked (either red or black indelible ink) and radioactively labeled Jeffrey pine seeds in a feeder near the center of the enclosure. We used scandium-46, a gammaemitting radionuclide with a half-life of 84.5 days, to label seeds (see Vander Wall 1992 for labeling procedure). Water, but no other food, was offered nearby. After $\sim 24$ hours, we returned and isolated the subject in the refuge bucket and then surveyed the enclosure with Geiger counters to locate cached seeds and the shells of eaten seeds. When we located a cache, we carefully excavated the seeds and mapped the precise location of each cache site.

We allowed each subject to search for cached seeds, during which time it could either recover its own caches or pilfer artificial caches. To reset the enclosure for the search phase of the experiment, we reburied 2 seeds $10 \mathrm{~mm}$ deep at each cache site (if some caches were close together or if the number of caches exceeded 30, then some caches were not reset). At $\sim 40 \mathrm{~cm}$ away from each rodent cache, we established a 2 nd cache (2 seeds at $10 \mathrm{~mm}$ deep) at a similar microsite. Microsite characteristics we considered included cover (under shrub or distance from shrub), substrate (mineral soil or plant litter), and distance to perennial plants (e.g., forbs, grass clumps) or objects (e.g., rocks, twigs). In these paired (artificial) caches, we used radioactively labeled seeds that differed in color (either black or red) from seeds in the real caches to avoid confusion between the 2 cache types. Seeds of both cache types had been previously handled by chipmunks. Humans never touched the seeds or the ground near the cache site; caches were dug with a stainless steel spoon, and seeds were handled only with forceps. At the beginning of each search phase, there was a 1:1 ratio of rodent caches to artificial caches. We covered caches carefully with soil and litter so that no evidence of digging was visible.

During the search phase of the experiment, it was necessary that rodents be able to detect the locations of all cache sites. Since olfaction 


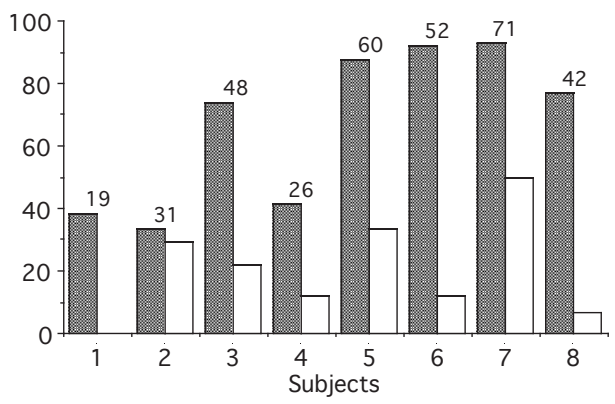

Fig. 1. The percentage of caches removed by each of 8 yellow-pine chipmunks. Shaded bars represent chipmunks' own caches; open bars represent paired artificial caches. Numbers above bars indicate the percentage of all caches removed during recovery trials.

can be limited under very dry conditions (Vander Wall 1995, 1998, 2000), we conducted cache recovery trials either in the spring when the soil was moist (2 trials) or during summer when we watered the enclosure with $175 \mathrm{~L}$ of water (6 trials). During summer trials, we also moistened seeds of both types (rodent caches and paired caches) before we reset caches to ensure that seeds were moist. Once caches were reset, we released the subject from the refuge bucket and gave it an opportunity to search for caches. Because we wanted to detect any difference in a subject's removal of their own versus paired caches during the search phase, we attempted to terminate the search trial after $25 \%-50 \%$ of caches had been located (after more than $50 \%$ of caches had been recovered, differences in removal of 1 cache type over the other would be obscured). Typically, we terminated search trials after $\sim 20$ hours. At that time, we released the subject and then checked all caches to determine which were intact and which were missing. We used the color of the seeds to avoid any confusion about which cache type (rodent cache or paired cache) had been removed. Data on the number of caches removed were analyzed using a paired $t$ test.

We also surveyed the entire enclosure to find any new caches. We compared the tendency of subjects to recache seeds from their own versus paired caches to see if subjects showed any tendency to conserve seeds that they had originally cached relative to those that they had pilfered. We used seed color to determine the origin of any seeds that the subjects had recached. We used a chi-squared test for independence to compare the number of "own" versus "pilfered" seeds removed from caches to the number of "own" and "pilfered" seeds recached.

\section{RESULTS}

Subjects made $24.0 \pm 4.0$ caches during the caching phase of the experiment and removed $43.8 \% \pm 17.7 \%$ of caches during the search phase. One subject removed fewer than the intended minimum of $25 \%$ (19\%), and 3 subjects removed more than the intended maximum of $50 \%(52 \%, 60 \%$ and $71 \%)$ of caches; however, the pattern of cache discovery of all subjects was unambiguous. Subjects removed $66.9 \% \pm 25.3 \%$ of their own caches and only $20.6 \% \pm 16.4 \%$ of the paired caches (paired $t$ test on numbers of caches removed: $t=4.737$, $\mathrm{df}=7, P<0.005)$. Seven of 8 subjects removed far more of their own caches than paired caches, but 1 chipmunk (subject 2) removed approximately equal numbers of its own and paired caches (Fig. 1).

Most (18 of 22 or $81.8 \%$ ) new caches that we discovered after search trials contained seeds that subjects had taken from their own caches. However, this was not significantly different from expected if we assume that seeds taken from own (133) and paired (41) caches were recached at random $\left(\chi^{2}=0.088, \mathrm{df}=1\right.$, $P>0.75)$.

\section{Discussion}

Since most subjects removed less than half of the caches available to them, the pattern of cache discovery they exhibited reveals how they were foraging at the beginning of the search phase. Previous studies have demonstrated that yellow-pine chipmunks will eventually find all of the caches in an enclosure when the soil is moistened (e.g., Vander Wall 1995). In a 2-choice experiment such as this, after half of the caches have been removed, subjects are then forced to search for the remaining caches and the subjects' foraging behavior may shift and their initial foraging mode may be obscured. Seven subjects retrieved mostly their own caches (Fig. 1). Since 3 of these subjects removed more than half of the caches available to them, it seems likely that the bias for recovering their own caches would have been even stronger if the search trials had been terminated earlier. 
The disproportionate recovery of one's own caches exhibited in this study suggests that subjects were foraging using spatial memory, an ability that has been demonstrated in yellow-pine chipmunks and other rodents (Jacobs and Liman 1991, Vander Wall 1991, Jacobs 1992). Most subjects also pilfered some paired caches. The moistened seeds were emitting odors (Vander Wall 2003), and subjects likely used those odors to find some paired caches, but most subjects did not appear to rely solely on olfactory cues to find caches. Had the scent of seeds been a more influential cue than spatial memory, chipmunks would have removed approximately equal numbers of their own caches and paired caches. One chipmunk (subject 2), however, did remove similar numbers of its own caches and artificial caches, suggesting that it had relied mostly on olfactory cues and apparently did not depend heavily on its spatial memory while foraging. It is unreasonable to assume that chipmunks were aware of paired caches but chose not to excavate them. Instead, it seems that chipmunks emptied all caches that they encountered, but that they encountered mostly their own caches. This result implies that chipmunks were not actively searching for the caches of other animals, but encountered some paired caches incidental to foraging for food on the ground surface or when moving about the enclosure.

Wrazen and Wrazen (1982) found that captive eastern chipmunks (Tamias striatus) preferred to eat food previously larder hoarded by conspecific chipmunks to food not previously handled by rodents. They found that eastern chipmunks did not exhibit a similar preference for food they themselves had stored. Wrazen and Wrazen (1982) attributed the ability of eastern chipmunks to determine previous ownership of food to "olfactory-gustatory cues" and attributed the preference to a component of the species' pilfering behavior. Yellow-pine chipmunks in this study showed no preference for artificial (i.e., simulated conspecific) caches. In our study, seeds were not stored in a larder, where odors of the owner are likely to be strong, but in small, scattered surface caches in soil where the odors of the cacher are likely to be much weaker. Our procedures did not incorporate the odors of competitors on seeds as a treatment effect, which would have permitted us to test this possibility directly; however, our methods did increase seed odors equally on subject and paired caches. Presumably, subjects could have used spatial memory to distinguish between their own and the artificial paired caches, which would have permitted them to preferentially pilfer artificial caches if they had been inclined to do so.

Our experiment demonstrated that yellowpine chipmunks did not forage indiscriminately for cached seeds. At least for the relatively short duration of the experiment $(<1$ day between caching and searching), chipmunks appeared to use their memories of past caching events to increase their efficiency in cache recovery. This is 1 way that food-storing animals can maintain a recovery advantage over naive individuals (Andersson and Krebs 1978, Vander Wall et al. 2006).

The results of this experiment are inconsistent with several previous studies that have found that yellow-pine chipmunks are very proficient and aggressive pilferers of the caches of other animals (Vander Wall 1998, 2000, Vander Wall and Jenkins 2003, Vander Wall et al. 2006). The results of this study suggest the opposite: that chipmunks recover their own caches before searching for caches to pilfer. Two things might help to reconcile these different results.

First, the artificial nature of the experimental arenas (e.g., small area, novel environment, disturbed cache sites) and the short time between caching and searching could have affected the chipmunk's mode of foraging. For example, we conducted trials during a year when no Jeffrey pine seeds were available. Chipmunks might have "known" that their Jeffrey pine seed (one of their preferred foods; Kuhn and Vander Wall in press) caches were the only ones present, so they did not look for the caches of other animals. If we could have conducted trials under more natural conditions, when Jeffrey pine seeds were abundant (mid-September to early November of most years), chipmunks might have used a different foraging mode aimed at pilfering the caches of other animals.

Second, one must consider how the foraging context differs between free-ranging chipmunks and chipmunks in our experimental enclosures. In the wild, yellow-pine chipmunks share space with dozens of conspecifics. This means that for every cache an individual makes, there are probably dozens of conspecific caches and even more caches if we include those made by 
other species (e.g., Vander Wall et al. 2001, Thayer and Vander Wall 2005). An individual's caches form only a small fraction of the available cached food within its home range. In this experiment the ratio of "own" caches to "other" caches was 1:1; in the field it could be 1:20 or more. Even if an individual chipmunk searches for its own caches, as demonstrated in this experimental study, it is foraging in an environment where its caches are greatly outnumbered by caches made by other animals. Thus, the time that a chipmunk spends searching for its own caches in a natural setting may be the same as that in our enclosures; however, the ratio of caches recovered to those pilfered would likely increase (compared to this study) simply as a result of conspecific cache density. The relative rarity of one's own caches, combined with longer periods of time and a home range much larger than the enclosure, probably results in more pilfering than cache recovery. More studies are needed to determine whether these ideas are correct; however, this study suggests that yellow-pine chipmunks search for their own caches but also suggests that this finding is not inconsistent with them being effective pilferers of the caches of other animals.

\section{Literature Cited}

Andersson, M., AND J. Krebs. 1978. On the evolution of hoarding behavior. Animal Behaviour 26:707-711.

Daly, M., L.F. Jacobs, M.I. Wilson, and P.R. Behrends. 1992. Scatter hoarding by kangaroo rats (Dipodomys merriami) and pilferage from their caches. Behavioral Ecology 3:102-111.

JACOBS, L.F. 1992. Memory for cache locations in Merriam's kangaroo rats. Animal Behaviour 43:585-593.

JACOBS, L.F., AND E.R. LIMAN. 1991. Grey squirrels remember the location of buried nuts. Animal Behaviour 41:103-110.

Kraus, B. 1983. A test of the optimal-density model for seed scatterhoarding. Ecology 64:608-610.

Kuhn, K.M., AND S.B. VANDER WaLL. In press. Linking summer foraging to winter survival in yellow pine chipmunks (Tamias amoenus). Behavioral Ecology.
Leaver, L.A., and M. DaLy. 2001. Food caching and differential cache pilferage: a field study of coexistence of sympatric kangaroo rats and pocket mice. Oecologia 128:577-584.

StAPANIAN, M.A., AND C.C. SMITH. 1978. A model for seed scatterhoarding: coevolution of fox squirrels and black walnuts. Ecology 59:884-896.

Thayer, T.C., AND S.B. VANDER WaLl. 2005. Interactions between Steller's jays and yellow pine chipmunks over scatter-hoarded sugar pine seeds. Journal of Animal Ecology 74:365-374.

VANDER WALL, S.B. 1991. Mechanisms of cache recovery by yellow pine chipmunks. Animal Behaviour 41: 851-863.

1992. The role of animals in dispersing a "winddispersed" pine. Ecology 73:614-621.

1995. Influence of substrate water on the ability of rodents to find buried seeds. Journal of Mammalogy $76: 851-856$.

1998. Foraging success of granivorous rodents: effects of variation in seed and soil water on olfaction. Ecology 79:233-241.

2000. The influence of environmental conditions on cache recovery and cache pilferage by yellow pine chipmunks (Tamias amoenus) and deer mice (Peromyscus maniculatus). Behavioral Ecology 11:544-549.

. 2003. How rodents smell buried seeds: a model based on the behavior of pesticides in soil. Journal of Mammalogy 84:1089-1099.

Vander Wall, S.B., J.S. Briggs, S.H. Jenkins, K.M. Kuhn, T.C. ThaYer, AND M.J. BECK. 2006. Do food-hoarding animals have a cache recovery advantage? Determining recovery of stored food. Animal Behaviour 72:189-197.

Vander Wall, S.B., and S.H. Jenkins. 2003. Reciprocal pilferage and the evolution of food-hoarding behavior. Behavioral Ecology 14:656-667.

Vander Wall, S.B., T.C. Thayer, J.S. Hodge, M.J. Beck, AND J.K. ROTH. 2001. Scatter-hoarding behavior of deer mice (Peromyscus maniculatus). Western North American Naturalist 61:109-113.

WraZen, J.A., AND L.A. WraZEN. 1982. Hoarding, body mass dynamics, and torpor components of the survival strategy of the eastern chipmunk. Journal of Mammalogy 63:63-72.

Received 27 September 2007 Accepted 11 February 2008 